
3 Research Square
Preprints are preliminary reports that have not undergone peer review.
They should not be considered conclusive, used to inform clinical practice,
or referenced by the media as validated information.

\title{
Dynamic Change of Pain Sensitisation at Acupoints in Patients with Knee Osteoarthritis and Disease Severity: A Multilevel Analysis of a Longitudinal Study
}

Guoyan Geng ( $\square$ ggyjanet@163.com )

Chengdu University of Traditional Chinese Medicine https://orcid.org/0000-0002-0730-2170

Yong Yu

Sichuan University

Mingsheng Sun

Chengdu University of TCM: Chengdu University of Traditional Chinese Medicine

Zihan Yin

Chengdu University of TCM: Chengdu University of Traditional Chinese Medicine

Jiao Chen

Chengdu University of TCM: Chengdu University of Traditional Chinese Medicine

Hui Zheng

Chengdu University of TCM: Chengdu University of Traditional Chinese Medicine

Dingju Cai

Chengdu University of TCM: Chengdu University of Traditional Chinese Medicine

Liaojun Luo

Chengdu University of TCM: Chengdu University of Traditional Chinese Medicine

Chunxia Yang

Sichuan University

Ling Zhao

Chengdu University of TCM: Chengdu University of Traditional Chinese Medicine

Fanrong Liang

Chengdu University of TCM: Chengdu University of Traditional Chinese Medicine

Research

Keywords: KOA, Acupoint, PPT, Longitudinal study, Disease severity

Posted Date: December 2nd, 2020

DOI: https://doi.org/10.21203/rs.3.rs-117502/v1

License: @ (1) This work is licensed under a Creative Commons Attribution 4.0 International License. Read Full License 


\section{Abstract}

Background and aim: Acupuncture alleviates pain and improves physical function in knee osteoarthritis (KOA). The therapeutic effect of acupuncture may depend on acupoint selection. This longitudinal study aimed to observe dynamic change of acupoint sensitization in knee osteoarthritis (KOA) patients, and to investigate the relationship between acupoint PPTs and disease severity.

Methods: Two-hundred-and-forty-six KOA patients were enrolled in this longitudinal study from 5 clinical centers.; data from 216 samples were analyzed. All participants underwent PPT assessment of 19 acupoints once weekly for 4 weeks. Multilevel analysis of repeated measurement data was performed.

Results: The PPTs at each acupoint decreased across the four timepoints, as the 19 acupoints became more pain-sensitive over time. Single-factor multilevel analysis showed a greater decrease in acupoint PPT at clinical stage $\geq$ III than clinical stage I ( $<<0.05)$; PPTs at Xuehai (SP-10), Heding (EX-LE2), Ququan (LR8), Yingu (KI-10), Xiguan (LR-7) and Qiuxu (GB-40) decreased more in imaging classification II than imaging classification I ( $<<0.05)$; PPT at Yaoyangguan (DU-3) decreased more in imaging classification $\geq I I I$ than imaging classification I $(p<0.05)$. Multi-factor multilevel analysis showed that the PPTs of Heding (EX-LE2), Liangqiu (ST-34), Ququan (LR-8), Dubi (ST-35), Weiyang (BL-39), Yinglingquan (SP-9), Xiguan (LR-7), Zusanli (ST-36), Yanglingquan (GB-34), Qiuxu (GB-40), and Weizhong (BL-40) decreased more with the progression of clinical stages ( $p<0.05)$; PPTs at Xuehai (SP-10), Heding (EX-LE2), Ququan (LR-8), Yingu (KI-10), Xiguan (LR-7), and Qiuxu (GB-40) decreased more in imaging classification II than imaging classification I ( $<<0.05)$; PPT at Qiuxu (GB-40) decreased more in imaging classification $\geq I I I$ than imaging classification I $(p<0.05)$.

Conclusion: The correlated acupoints became more pain-sensitive over time, and the acupoint PPTs were in accordance with disease severity. Liangqiu (ST34), Dubi (ST-35), Weiyang (BL-39), Yinglingquan (SP-9), Xiguan (LR-7), Zusanli (ST-36), Yanglingquan (GB-34), and Qiuxu (GB-40) were most related to disease severity, they should be recommended clinically.

Trial registration: ChiCTR, ChiCTR1800014616. Registered 24 January 2018 - Retrospectively registered, http://www.chictr.org.cn/showproj.aspx?proj=24037

\section{Article Summary}

\section{Strengths and limitations of this study}

- To our knowledge, this is the first longitudinal study to observe the dynamic change of acupoint sensitization in knee osteoarthritis (KOA) patients.

- Multilevel model analyses were used to comprehensively explore the relationships between various factors and acupoint sensitization.

- A limitation of this study is that 4 weeks might be not long enough to observe pathological changes.

- Although acupuncture treatment and some pharmacotherapies were not allowed during the 4-week observation period, other pharmacotherapy or nonpharmacotherapy treatments (except for acupuncture) and self-adjustment were not recorded, which might lead to certain bias.

\section{Background}

Osteoarthritis $(\mathrm{OA})$ is one of the most common musculoskeletal disorders and is a leading cause of pain and disability worldwide [1], which represents a substantial and increasing health burden with notable implications for the individuals affected, healthcare systems, and wider socioeconomic costs [2-4]. Clinically, the knee is the most common site of OA.[5] In the United States, symptomatic knee OA (KOA) affects approximately 9.9 million adults.[6] In China, the prevalence of symptomatic KOA is about $8.1 \%$, and is higher in women (10.3\%) than in men (5.7\%). [7] About $25 \%$ of adults > 55 years old experience significant knee pain, half of whom have radiographic OA changes and a quarter of whom have significant disability.[8] The reported risk factors for KOA include age, female sex, being overweight, genetics, and repeated knee bending or heavy lifting.[1,6] With the combined effects of ageing and increasing obesity in the global population, along with increasing numbers of joint injuries, this already burdensome syndrome is becoming more prevalent.

The most disabling symptom experienced by patients with OA is pain, which is a major driver of clinical decision-making and health service use, and is best framed within a biopsychosocial model.[1] Effective pain control is needed to improve the quality of life and minimize disability of patients with KOA. Current guidelines recommend first-line treatment comprising non-pharmacological methods such as education and self-management, exercise, weight loss if overweight or obese, and walking aids as indicated. $[6,9,10]$ Management of OA pain is based on a sequential hierarchical approach; once the first-line treatment becomes inadequately effective, pharmacological methods become the main form of treatment.[11, 12] Non-steroidal anti-inflammatory drugs and paracetamol are most often recommended as first-line analgesics for KOA.[6, 9, 13] In individual patients, the benefit of oral non-steroidal anti-inflammatory drugs has to be weighed against their potential adverse effects on the gastrointestinal, cardiovascular, and hepatic systems.[14, 15]

Acupuncture has acquired an increasing amount of attention as an alternative therapy for pain management, [16-18] with long-term effects and low risk of adverse events. $[19,20]$ Evidence indicates that acupuncture alleviates pain and improves the physical function of patients with KOA, with a low risk of adverse reactions.[21-24]. However, some studies have reported that acupuncture has no beneficial effects for KOA.[25, 26] This difference in therapeutic effect may be caused by variation in the acupoints treated, as the appropriate selection of acupoints is crucial to the achievement of the treatment effect.[27] Individualized acupoint prescription is the main method of acupoint selection in clinical practice, and the acupoint prescription should be adapted in accordance with the changes in condition. According to the theory of traditional Chinese medicine (TCM), there are connections between the disease conditions and their respective points (i.e., traditional acupoints and tender points on the body surface).[28] These points become sensitized when the body is in a diseased state, and stimulation of the sensitive points leads to an improvement of the disease conditions. [29, 30]

Pain sensitization at acupoints has been observed in patients with gastric ulcers or gastritis,[31] stable angina pectoris,[32] shoulder pain,[33] and KOA[34]. The pain sensitization of acupoints is assessed using the pressure pain threshold (PPT),[35]which is a semi-objective method used to quantify localized pain. 
$[33,36,37]$ The present longitudinal study with multilevel modelling analysis was conducted to determine the optimal acupoint prescription in KOA, investigate the dynamic changes over time in acupoint pain sensitization in patients with $\mathrm{KOA}$, and evaluate the impact of disease severity on acupoint pain sensitization.

\section{Methods}

\section{Study design}

This is a longitudinal study. The protocol was developed in accordance with the Strengthening the Reporting of Observational Studies in Epidemiology guidelines.[38] This study was designed in accordance with the principles of the Declaration of Helsinki. The study protocol has been approved, and is registered on the primary registry in the WHO registry network (Chinese Clinical Trial Registry: no. ChiCTR1800014616).

\section{Participants}

Patients with KOA were recruited from March 2017 to November 2018 at the outpatient clinics of the Departments of Acupuncture and Moxibustion, and Orthopaedics in five clinical centers in China: Chengdu University of Traditional Chinese Medicine, Hunan University of Traditional Chinese Medicine, Shaanxi University of Traditional Chinese Medicine, Shanxi University of Traditional Chinese Medicine, and Guizhou University of Traditional Chinese Medicine.

The diagnosis of KOA was made in accordance with the criteria in the Guidelines for the Medical Management of Osteoarthritis. [39-41]

Inclusion criteria

Patients were eligible for study inclusion if they: (1) met the KOA diagnostic criteria; (2) were 40-80 years old; (3) provided written informed consent for all study procedures.

\section{Exclusion criteria}

Patients were excluded if they met any of the following criteria: (1) diagnosis of conditions leading to skeletal disorders, such as tuberculosis, tumors or rheumatism of the knee joint, and rheumatoid arthritis; (2) sprain or trauma in the lower limbs; (3) inability to walk properly due to foot deformity or pain; (4) inability to answer the questionnaire due to mental disorders and/or intellectual disability; (5) concomitant severe cardiovascular disease, liver and kidney function impairment, immune deficiency, diabetes mellitus, or hemopathy.

\section{Protocol}

All participants completed a number of questionnaires, including demographic data (age, sex, race, height, and weight) and disease information (disease duration and TCM syndrome differentiation). Clinical stage was diagnosed by professional clinicians in accordance with clinical symptoms and radiographic results.[42,43] After informed consent was obtained, the patients were assessed to determine their eligibility in accordance with the inclusion and exclusion criteria. PPT measurements were carried out once a week for 4 weeks for all included patients.

\section{PPT measurement}

Based on literature data-mining and a pilot study[34], we identified the 19 most frequently used acupoints in the treatment of KOA. The affected knee was exposed to enable the marking of 15 acupoints on the lower limb, and the back was exposed to enable the marking of the other four acupoints (Mingmen (DU4), Yaoyangguan (DU-3), Shenshu (BL-23), and Dazhu (BL-11)). The procedure was carried out by expert acupuncturists, as performed in our previous study [37]. The acupuncturist used the FDIX Force Gauge (Force One FDIX, Wagner Instruments, Greenwich, Connecticut, USA) to make two measurements of the PPT at each of the 19 acupoints. All participants were instructed to indicate when the pressure became painful; at that time, the pressure was immediately stopped, and the force was recorded. The PPT was calculated as the mean of two measurements at each point. If there was more than 500 gf difference between the two PPT measurements at one acupoint, the PPT of the acupoint was measured a third time. There was an interval of approximately 2 minutes between measurements.

\section{Sample size calculation}

As this study was a longitudinal repeated measurement study, the longitudinal change rate was applied for the sample size estimation.[44] According to the previous literature, the longitudinal change rate of acupoint sensitization ranges from $10-80 \% .[45,46]$ In the present study, it was expected that the change rate of acupoint sensitization in the cohort would be $30 \%$, and the tender point sensitization of the cohort would be $5 \%$. Based on the estimation of variance of a random cross-section, where the slope was 24 , residual value was $10, a=0.05$, and $\beta=0.9$, the minimum required sample size was 233 . Assuming a $10 \%$ loss rate, the final sample size was set as 256.

\section{Data analysis}

\section{Routine statistical analysis}

Data were inputted into a computer with Epidata 3.0 software and analyzed by a statistical software package (SAS 9.4). The quantitative data were described by mean \pm standard deviation; the qualitative data were described by the frequency and percentage. Descriptive analysis was used to describe the basic patient characteristics and disease severity. 
Acupoint PPT data has a distinct hierarchical structure, with aggregation in different districts and patients. In consideration of the auto-correlation between the data and the difficulty of repeated measurement variance analysis when there are missing data, the repeated measurement multilevel model was used to analyze the relationships between the severity of the disease (based on clinical stage or imaging classification), time, and acupoint PPT. The PPT changes at the 19 acupoints were analyzed by multistage multiple regression. A three-level random intercept regression model was constructed, with timepoint at level 1 , patient at level 2, and district at level 3. Subsequently, the multilevel multiple regression models included seven independent variables, comprising six on the patient level and one on the timepoint level. The six patient-level variables were age (in years), sex, body mass index (BMI), disease severity, disease duration (in years), and TCM syndrome differentiation. The timepoint-level variable was measurement time (in weeks). All continuous variables were centered (subtracted from the mean) before the analysis. Multilevel analysis of repeated measurement data was performed by MLwiN 2.30 software. The significance level was set at 0.05 .

Table 1 shows the encoding and assignment of each variable in the original data. To investigate the relationship between acupoint PPTs and disease severity in patients with KOA, the PPTs of the 19 acupoints in the four follow-up surveys was taken as the dependent variable. The level of significance was set at 0.05 . The ordered multi-classification variables (clinical stage/ imaging classification) were included in the form of grouped linear variables and dummy variables, respectively, and model I and model II were fitted to determine whether the difference in negative twice logarithmic likelihood between the two models was statistically significant; the clinical stage was finally included in the form of a dummy variable.

Table 1

Variable coding and assignment of the original data

\begin{tabular}{|c|c|c|}
\hline Indicator & Variable & State variable \\
\hline District (level 3) & Region & $1 \rrbracket 5$ \\
\hline Patient (level 2) & ID & $1 \otimes 216$ \\
\hline Time point (level 1) & NO & $1 \otimes 864$ \\
\hline Time & Time & $0 \rrbracket 3$ \\
\hline Age & Age & $43 \llbracket 75$ \\
\hline Gender & Gender & $0=$ male, $1=$ female \\
\hline Body Mass Index & $\mathrm{BMI}$ & $17.19 \llbracket 32.42$ \\
\hline \multirow[t]{3}{*}{ Clinical stage } & \multirow[t]{3}{*}{ Clinical } & $0=$ Clinical stage I \\
\hline & & 1 = Clinical stage II \\
\hline & & 2 = Clinical stage III and above \\
\hline \multirow[t]{3}{*}{ Imaging classification } & \multirow[t]{3}{*}{ Imaging } & $0=$ Imaging classification I \\
\hline & & 1 = Imaging classification II \\
\hline & & 2 = Imaging classification III and above \\
\hline Duration of disease & Duration & $0.8 \sim 40$ \\
\hline \multirow[t]{3}{*}{ Syndrome differentiation of TCM } & \multirow[t]{3}{*}{ Syndrome } & $0=$ Deficiency of liver and kidney \\
\hline & & $1=$ Yang deficiency with congealing cold \\
\hline & & 2 = Qi stagnation and blood stasis \\
\hline
\end{tabular}

\section{Results}

\section{Recruitment and baseline characteristics}

From March 2017 to November 2018, 246 patients from five clinical centers were enrolled, and data from 216 samples were analyzed. The characteristics and disease information of these 216 patients are shown in Table 2. 
Table 2

Patient characteristics

\begin{tabular}{|ll|}
\hline characteristics & No. (\%) \\
\hline Clinical center & 60 \\
Chengdu University of TCM, & 36 \\
Hunan University of TCM & 29 \\
Shaanxi University of TCM & 44 \\
Shanxi University of TCM & 47 \\
\hline & \\
\hline Guizhou University of TCM & \\
\hline Age, mean (SD), y & $57.23(7.67)$ \\
\hline Gender & 175 \\
\hline Female & 41 \\
\hline Male & $23.79(2.78)$ \\
\hline BMI, mean (SD) & $5.35(6.20)$ \\
\hline Duration of illness, mean (SD), y & \\
\hline bValues are reported as number (\%) unless otherwise indicated. \\
\hline
\end{tabular}

Longitudinal comparison of the four PPT measurements at the selected acupoints

The four PPTs at each acupoint showed a decreasing trend, as the 19 acupoints became more pain-sensitive with time (Fig. 1). With the progression of the disease, the acupoints associated with KOA became more pain-sensitized.

\section{Multilevel statistical analysis}

Single-factor multilevel model

Single-factor multilevel model analysis showed that the acupoint PPTs differed in accordance with the clinical stage (Table 3 ) and imaging classification (Table 4). Compared with patients at clinical stage I, the PPTs of each acupoint were significantly lower in patients at clinical stage III and above $(p<0.05)$. Compared with patients with imaging classification I, the PPTs at Xuehai (SP-10), Heding (EX-LE2), Ququan (LR-8), Yingu (KI-10), Xiguan (LR-7), and Qiuxu (GB-40) were significantly lower in patients with imaging classification II $(p<0.05)$; the PPT at Yaoyangguan (DU-3) were significantly lower in patients with imaging classification III and above compared with patients with imaging classification I $(p<0.05)$. 
Table 3

Single-factor multilevel model of acupoint PPT and Clinical stage

\begin{tabular}{|lll|}
\hline Acupoints & Variable & \\
\cline { 2 - 3 } & Clinical stage(II:I) & Clinical stage(III:I) \\
\hline Xuehai(SP-10) & $38.88(159.72)$ & $-512.20(245.95)^{*}$ \\
\hline Heding(EX-LE2) & $-32.93(225.77)$ & $-1025.18(348.67)^{*}$ \\
\hline Liangqiu(ST-34) & $-181.60(214.37)$ & $-1025.70(329.01)^{*}$ \\
\hline Ququan(LR-8) & $-0.22(168.77)$ & $-656.66(260.21)^{*}$ \\
\hline Yingu(KI-10) & $-118.28(199.72)$ & $-679.81(318.59)^{*}$ \\
\hline Neixiyan(EX-LE5) & $-130.08(242.16)$ & $-693.42(372.90)$ \\
\hline Dubi(ST-35) & $-0.61(195.43)$ & $-747.39(301.91)^{*}$ \\
\hline Yinglingquan(SP-9) & $-73.96(172.46)$ & $-538.14(265.67)^{*}$ \\
\hline Xiguan(LR-7) & $-30.18(172.01)$ & $-707.89(264.28)^{*}$ \\
\hline Zusanli(ST-36) & $-238.38(228.83)$ & $-918.35(353.26)^{*}$ \\
\hline Yanglingquan(GB-34) & $-165.05(210.25)$ & $-700.77(324.53)^{*}$ \\
\hline Weiyang(BL-39) & $-169.44(194.36)$ & $-785.96(298.64)^{*}$ \\
\hline Weizhong(BL-40) & $-75.26(203.63)$ & $-795.74(312.81)^{*}$ \\
\hline Xuanzhong (GB-39) & $-92.73(220.52)$ & $-771.11(341.48)^{*}$ \\
\hline Qiuxu (GB-40) & $-240.18(189.50)$ & $-753.51(292.52)^{*}$ \\
\hline Dazhu(BL-11) & $25.53(221.07)$ & $-637.82(342.45)^{*}$ \\
\hline Shenshu(BL-23) & $-74.48(248.74)$ & $-828.90(386.04)^{*}$ \\
\hline Mingmen(DU-4) & $-56.03(220.17)$ & $-677.06(342.79)$ \\
\hline Yaoyangguan(DU-3) & $-110.94(218.06)$ & $-789.12(338.85)^{*}$ \\
\hline 0.05 (statistical significance). & \\
\hline
\end{tabular}


Table 4

Single-factor multilevel model of acupoint PPT and Imaging classification

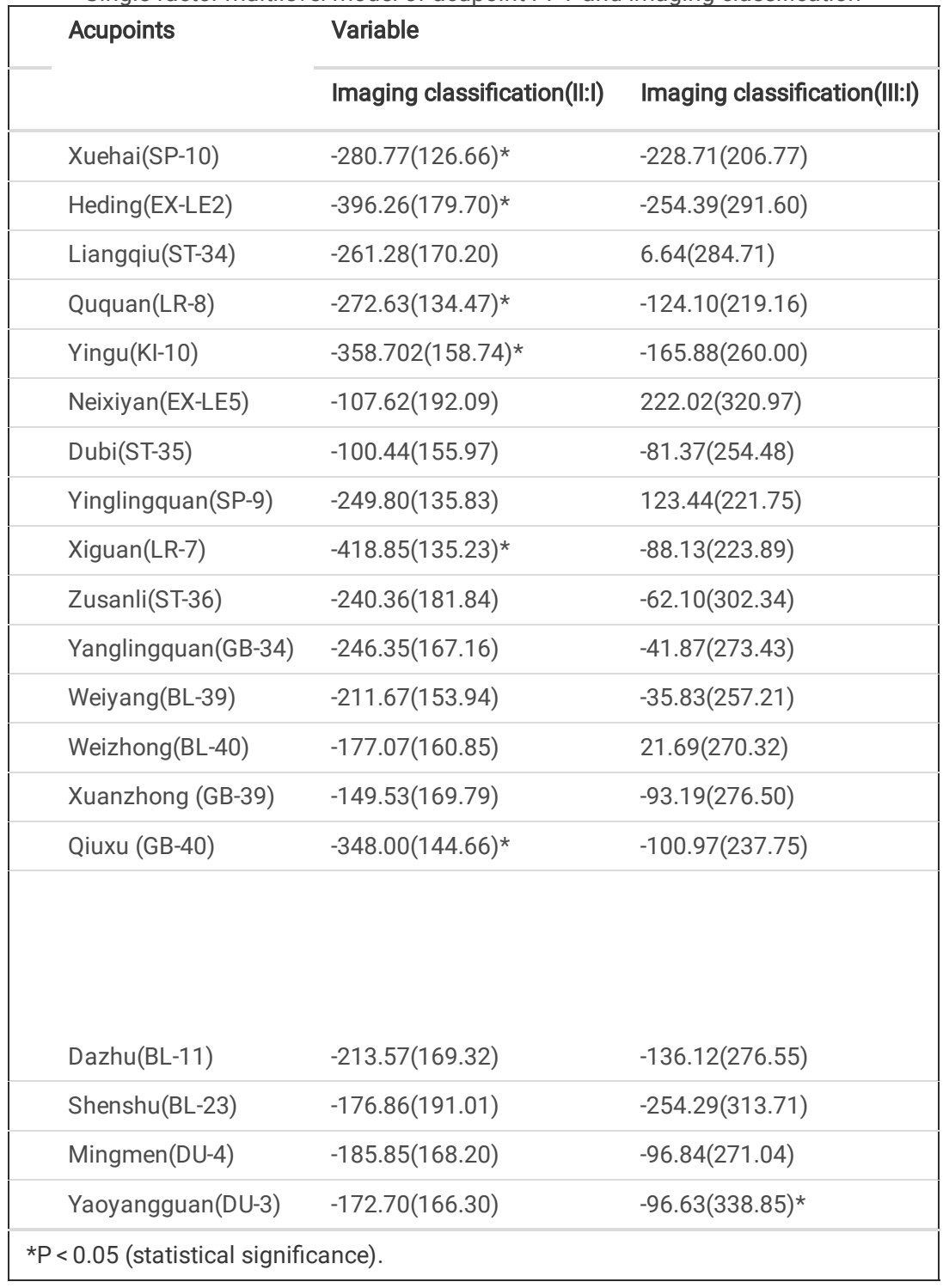

Multi-factor multilevel model analysis

To explore the factors influencing acupoint PPT in patients with KOA, the PPT of 19 acupoints was taken as the dependent variable, and the factors that showed significance $(P<0.1)$ in the single-factor multilevel model (sex, measurement time, $\mathrm{BMI}$, and clinical stage/imaging classification) were entered in the multi-factor multilevel model of repeated measurement data, with the level of significance set at 0.05 .

The random effects results in Table 5 showed that the acupoint PPTs differed between the 216 individuals, and the PPTs also differed between the acupoints within each individual. With the other factors kept constant, the acupoint PPTs decreased with time, showing that the pain sensitivity of the acupoints increased. Each patient had different slope changes with time at the following 11 acupoints: Xuehai (SP-10), Liangqiu (ST-34), Neixiyan (EX-LE5), Dubi (ST-35), Weiyang (BL-39), Yinglingquan (SP-9), Xiguan (LR-7), Zusanli (ST-36), Yanglingquan (GB-34), Xuanzhong (GB-39), and Qiuxu (GB-40) ( $p<0.05)$. 
Table 5

a. Random coefficient model of the factors affecting the PPTs of the acupoints

\begin{tabular}{|c|c|c|c|c|c|c|c|c|c|c|}
\hline \multirow[t]{2}{*}{ Variable } & \multicolumn{10}{|c|}{ Acupuncture points } \\
\hline & Xuehai & Heding & Liangqiu & Ququan & Yingu & Nexiyan & Dubi & Yinlingquan & Xiguan & Zusanli \\
\hline \multicolumn{11}{|l|}{$\begin{array}{l}\text { Fixed } \\
\text { effect }\end{array}$} \\
\hline \multirow[t]{2}{*}{ Intercept } & 2630.29 & 3419.18 & 3314.14 & 2524.83 & 2726.1 & 3263.2 & 2961.57 & 2567.66 & 2370.72 & 3321.27 \\
\hline & $(276.33)^{\star}$ & $(354.40) \star$ & $(348.78)^{\star}$ & $(287.20)^{\star}$ & $(300.00)^{*}$ & $(327.29)^{\star}$ & $(295.65)^{\star}$ & $(287.69)^{\star}$ & $(291.10)^{\star}$ & $(331.82)^{\star}$ \\
\hline \multirow[t]{2}{*}{ Time } & -111.79 & -155 & -144.86 & -94.6 & -71.42 & -190.81 & -158.34 & -100.52 & -89.59 & -170.56 \\
\hline & $(18.52)^{\star}$ & $(26.75)^{\star}$ & $(21.75)^{\star}$ & $(19.50)^{\star}$ & $(22.69)^{\star}$ & $(27.94)^{\star}$ & $(25.53)^{\star}$ & $(20.77)^{\star}$ & $(18.64)^{\star}$ & $(27.36)^{\star}$ \\
\hline \multirow[t]{2}{*}{ Gender } & -422.49 & -615.65 & -700.18 & -401 & -383.61 & -563.31 & -326.72 & -420.59 & -256.96 & -461.5 \\
\hline & $(126.06)^{\star}$ & $(179.90)^{\star}$ & $(175.70)^{\star}$ & $(134.59)^{\star}$ & $(164.79)^{\star}$ & $(188.29)^{\star}$ & $(159.46)^{\star}$ & $(136.88)^{\star}$ & -137.21 & $(178.07)^{\star}$ \\
\hline \multirow[t]{2}{*}{ BMI } & 21.62 & 45.27 & 44.22 & 32.72 & 49.19 & 49.58 & 26.98 & 25.5 & 36.24 & 58.53 \\
\hline & (17.39) & $(24.79)$ & $(24.27)$ & $(18.57)$ & $(22.72)^{\star}$ & $(25.97)$ & (21.99) & (18.88) & (18.92) & $(24.56)^{\star}$ \\
\hline \multicolumn{11}{|l|}{$\begin{array}{l}\text { Clinical } \\
\text { stages }\end{array}$} \\
\hline \multirow[t]{2}{*}{ II:I } & 99.86 & 13.54 & -115.47 & 31.69 & -71.91 & -47.57 & 18.92 & -2.77 & -69.42 & -215.25 \\
\hline & (151.32) & $(215.61)$ & $(206.56)$ & (161.39) & (196.7) & (219.88) & (190.73) & (163.65) & (161.75) & $(209.94)$ \\
\hline \multirow[t]{2}{*}{ |II: | } & -458.21 & -1059.73 & -953.92 & -576.57 & -582.76 & -670.56 & -778.83 & -513.6 & -809.67 & -896.86 \\
\hline & $(236.62)$ & $(338.20)^{*}$ & $(320.46)^{\star}$ & $(252.63)^{\star}$ & (316.37) & (346.6) & $(298.41)^{\star}$ & $(256.55)^{\star}$ & $(253.51)^{\star}$ & $(332.82)^{\star}$ \\
\hline \multicolumn{11}{|l|}{$\begin{array}{l}\text { Random } \\
\text { effect }\end{array}$} \\
\hline \multirow{2}{*}{$\begin{array}{l}\sigma_{\mathrm{u} 1}^{2}(\mathrm{Time} \\
\text { slope) }\end{array}$} & 19031.92 & 29543.26 & 25337.12 & 14592.19 & 10076.6 & 87102.37 & 64008.37 & 32960.06 & 21746.71 & 83297 \\
\hline & $(8049.07)^{\star}$ & $(17112.5)$ & $(11102.85)^{\star}$ & (9132.58) & (12682.63) & $(17122.44)^{*}$ & $(14487.15)^{\star}$ & $(9837.81)^{\star}$ & $(8052.66)^{\star}$ & $(16411.73)^{y}$ \\
\hline
\end{tabular}


Table 5

b. Random coefficient model of the factors affecting the PPTs of the acupoints

\begin{tabular}{|c|c|c|c|c|c|c|c|c|c|}
\hline \multirow[t]{2}{*}{ Variable } & \multicolumn{9}{|c|}{ Acupuncture points } \\
\hline & Yanglingquan & Weiyang & Weizhong & Xuanzhong & Qiuxu & Dazhu & Shenshu & Mingmen & Yaoyangguan \\
\hline \multicolumn{10}{|l|}{ Fixed effect } \\
\hline \multirow[t]{2}{*}{ Intercept } & 3181.79 & 2759.81 & 2701.76 & 2116.65 & 1384.25 & 2324.13 & 2451.87 & 3311 & 3355.98 \\
\hline & $(329.84)^{\star}$ & $(308.05)^{\star}$ & $(312.65)^{\star}$ & $(645.97)^{\star}$ & $(574.48)^{\star}$ & $(651.74)^{\star}$ & $(716.37)^{*}$ & $(300.20)^{\star}$ & $(303.32)^{\star}$ \\
\hline \multirow[t]{2}{*}{ Time } & -146.6 & -69.98 & -61.48 & -180.21 & -107.57 & -123.38 & -159.09 & -131.39 & -97.42 \\
\hline & $(27.15)^{\star}$ & $(21.94)^{*}$ & $(20.31)^{\star}$ & $(24.91)^{*}$ & $(21.38)^{\star}$ & $(22.70)^{\star}$ & $(26.37)^{\star}$ & $(24.05)^{\star}$ & $(23.11)^{*}$ \\
\hline \multirow[t]{2}{*}{ Gender } & -532.61 & -412.03 & -425.55 & -445.92 & -209.72 & -430.25 & -478.63 & -456.73 & -515.55 \\
\hline & $(171.05)^{\star}$ & $(162.25)^{\star}$ & $(170.77)^{\star}$ & $(170.87)^{\star}$ & -146.93 & $(173.60)^{*}$ & $(193.42)^{\star}$ & $(173.25)^{\star}$ & $(173.57)^{\star}$ \\
\hline \multirow[t]{2}{*}{ BMI } & 43.72 & 51.92 & 55.72 & 40.91 & 49.06 & 32.2 & 43.17 & 43.56 & 25.52 \\
\hline & $(23.64)$ & $(22.40)^{\star}$ & $(23.59)^{\star}$ & $(23.52)$ & $(20.20)^{\star}$ & $(23.89)$ & $(26.62)$ & $(23.83)$ & $(23.87)$ \\
\hline \multicolumn{10}{|l|}{ Clinical stages } \\
\hline \multirow[t]{2}{*}{$\|: 1$} & -112.32 & -126.46 & -30.88 & 20.23 & -159.39 & 122.66 & 55.99 & -0.15 & -31.53 \\
\hline & (204.99) & (191.45) & $(200.04)$ & $(211.49)$ & (181.98) & $(214.14)$ & $(238.11)$ & (213.98) & $(214.2)$ \\
\hline \multirow[t]{2}{*}{ |II:I } & -711.11 & -700.97 & -749.02 & -597.25 & -669.35 & -454.24 & -595.31 & -521.05 & -614.58 \\
\hline & $(319.53)^{\star}$ & $(296.80)^{*}$ & $(310.26)^{\star}$ & (331.28) & $(284.00)^{\star}$ & (335.12) & (373.78) & (335.73) & (335.34) \\
\hline \multicolumn{10}{|l|}{ Random effect } \\
\hline \multirow{2}{*}{$\sigma_{\mathrm{u} 1}^{2}$ (Time slope) } & 75850.52 & 42214.5 & 14551.09 & 41637.15 & 41492.53 & 12191 & 29237.11 & 8566.73 & 1710.59 \\
\hline & $(16304.22)^{\star}$ & $(10828.78)^{*}$ & (9937.19) & $(14160.62)^{\star}$ & $(10133.94)^{\star}$ & $(12516.7)$ & (16449.92) & (14246.73) & (13405.98) \\
\hline
\end{tabular}

The fixed effects results in Table 5 showed that the main factors related to acupoint PPTs in patients with KOA were sex, BMI, and clinical stage. Except for Qiuxu (GB-40) and Xiguan (LR-7), the PPTs of the other 17 acupoints were lower in females than males. The PPTs of Yingu (KI-10), Weiyang (BL-39),

Weizhong (BL-40), Qiuxu (GB-40), and Zusanli (ST-36) increased with the increase in BMI. The PPTs of Heding (EX-LE2), Liangqiu (ST-34), Ququan (LR-8), Dubi (ST-35), Weiyang (BL-39), Yinglingquan (SP-9), Xiguan (LR-7), Zusanli (ST-36), Yanglingquan (GB-34), Qiuxu (GB-40), and Weizhong (BL-40) decreased with the progression of clinical stage and disease severity, showing that the pain sensitivity of the acupoints increased. The acupoint PPTs of patients at clinical stage II tended to be lower than those of patients at clinical stage I, but this difference was not statistically significant. The acupoint PPTs of patients at clinical stage III were lower than those of patients at clinical stage I $(p<0.05)$.

The fixed effects results in Table 6 showed that the imaging classification of patients with KOA was related to the PPT of some acupoints. Compared with patients with imaging classification I, the PPTs at Xuehai (SP-10), Heding (EX-LE2), Ququan (LR-8), Yingu (KI-10), Xiguan (LR-7), and Qiuxu (GB-40) were significantly lower than those in patients with imaging classification II $(p<0.05)$; the PPTs at Qiuxu (GB-40) were significantly lower in patients with imaging classification III and above $(p<0.05)$. Furthermore, the PPT decreases over the 4-week observation period were significantly greater in patients with imaging classification II or $\geq$ III than in patients with imaging classification I $(p<0.05)$. 
Table 6

a. Random coefficient model of the factors affecting the PPTs of the acupoints

\begin{tabular}{|c|c|c|c|c|c|c|c|c|c|c|}
\hline \multirow[t]{2}{*}{ Variable } & \multicolumn{10}{|c|}{ Acupuncture points } \\
\hline & Xuehai & Heding & Liangqiu & Ququan & Yingu & Nexiyan & Dubi & Yinlingquan & Xiguan & Zusar \\
\hline \multicolumn{11}{|c|}{ Fixed effect } \\
\hline \multirow[t]{2}{*}{ Intercept } & 2229.01 & 2491.48 & 2214.46 & 1811.45 & 1603.42 & 2011.01 & 2297.51 & 1961.31 & 1558.16 & 1751. \\
\hline & $(508.16)^{\star}$ & $(702.26)^{\star}$ & $(680.60)^{\star}$ & $(536.89)^{\star}$ & $(627.21)^{\star}$ & $(702.07)^{*}$ & $(606.08)^{\star}$ & $(536.30)^{*}$ & $(540.99)^{\star}$ & $(672.4$ \\
\hline \multirow[t]{2}{*}{ Time } & -112.78 & -154.03 & -145.74 & -96.69 & -72.84 & 191.64 & -160.45 & -101.74 & -90.08 & -173.1 \\
\hline & $(18.47)^{\star}$ & $(26.87)^{\star}$ & $(21.68)^{\star}$ & $(19.48)^{\star}$ & $(22.67) \star$ & $(28.06) *$ & $(25.60)^{\star}$ & $(20.74)^{\star}$ & $(18.57)^{\star}$ & $(27.4 C$ \\
\hline \multirow[t]{2}{*}{ Gender } & -456.63 & -693.31 & -783.13 & -444.06 & -433.41 & -633.61 & -392.19 & -470.62 & -328.93 & -548.5 \\
\hline & $(124.13)^{\star}$ & $(177.66)^{\star}$ & $(174.28)^{\star}$ & $(132.76)^{*}$ & $(162.75)^{\star}$ & $(186.35)^{\star}$ & $(158.41)^{\star}$ & $(133.03)^{*}$ & $(133.70)^{\star}$ & $(175 . c$ \\
\hline \multirow[t]{2}{*}{$\mathrm{BMI}$} & 28.01 & 50.93 & 49.04 & 38.77 & 55.86 & 53.50 & 32.10 & 31.92 & 43.23 & 67.37 \\
\hline & (17.54) & $(25.07)^{\star}$ & $(24.61)^{\star}$ & $(18.75)^{\star}$ & $(22.98) \star$ & $(26.31)^{*}$ & $(22.35)$ & (18.79) & (18.88) & $(24.72$ \\
\hline \multicolumn{11}{|c|}{$\begin{array}{l}\text { Imaging } \\
\text { classifications }\end{array}$} \\
\hline \multirow[t]{2}{*}{$\|: \mid$} & -247.60 & -373.31 & -226.74 & -254.05 & -358.91 & -89.27 & -104.91 & -230.24 & -375.80 & -273.2 \\
\hline & $(118.72)^{*}$ & $(170.57)^{\star}$ & $(161.76)$ & $(127.14)^{\star}$ & $(154.68)^{\star}$ & (173.13) & $(150.25)$ & (127.37) & $(126.19) \star$ & (164.1 \\
\hline \multirow[t]{2}{*}{ III:I } & -157.62 & -216.10 & 76.52 & -48.17 & -129.34 & 235.12 & -67.29 & 192.28 & -22.97 & -46.11 \\
\hline & (192.68) & (274.99) & $(268.77)$ & (205.54) & (251.79) & (287.74) & $(245.09)$ & (206.03) & $(206.60)$ & $(270.5$ \\
\hline \multicolumn{11}{|l|}{$\begin{array}{l}\text { Random } \\
\text { effect }\end{array}$} \\
\hline \multirow{2}{*}{$\begin{array}{l}\sigma_{\mathrm{u} 1}^{2}(\mathrm{Time} \\
\text { slope })\end{array}$} & 197833.27 & 28218.16 & 23761.67 & 13937.22 & 10203.32 & 87373.78 & 63386.83 & 31283.03 & 19675.79 & $8340 \epsilon$ \\
\hline & $(8001.44)^{\star}$ & (17234.05) & $(11032.92)^{\star}$ & (9091.91) & (12596.14) & $(17199.21)^{\star}$ & $(14524.02)^{\star}$ & $(9812.26)^{\star}$ & $(8021.39) *$ & (1638 \\
\hline
\end{tabular}


Table 6

b. Random coefficient model of the factors affecting the PPTs of the acupoints

\begin{tabular}{|c|c|c|c|c|c|c|c|c|c|}
\hline \multirow[t]{2}{*}{ Variable } & \multicolumn{9}{|c|}{ Acupuncture points } \\
\hline & Yanglingquan & Weiyang & Weizhong & Xuanzhong & Qiuxu & Dazhu & Shenshu & Mingmen & Yaoyangguan \\
\hline \multicolumn{10}{|l|}{ Fixed effect } \\
\hline \multirow[t]{2}{*}{ Intercept } & 2083.38 & 1447.49 & 1385.11 & 2068.75 & 1346.10 & 2391.99 & 2470.41 & 2170.10 & 2618.14 \\
\hline & $(657.04)^{\star}$ & $(619.19)^{\star}$ & $(648.88)^{\star}$ & $(644.85)^{\star}$ & $(575.88)^{\star}$ & $(653.26)^{\star}$ & $(716.35)^{\star}$ & $(637.16)^{\star}$ & $(639.47)^{\star}$ \\
\hline \multirow[t]{2}{*}{ Time } & -150.21 & -70.11 & -62.04 & -180.24 & -106.78 & -123.67 & -157.99 & -128.29 & -93.97 \\
\hline & $(26.99)^{\star}$ & $(22.06)^{\star}$ & $(20.29) \star$ & $(25.05)^{\star}$ & $(21.39)^{\star}$ & $(22.87)^{\star}$ & $(26.50)^{\star}$ & $(24.12)^{\star}$ & $(23.19)^{\star}$ \\
\hline \multirow[t]{2}{*}{ Gender } & -590.74 & -475.57 & -485.17 & -497.22 & -265.87 & -467.02 & -522.96 & -511.70 & -579.73 \\
\hline & $(169.09)^{\star}$ & $(160.82)^{*}$ & $(168.93)^{\star}$ & $(167.87)^{\star}$ & (144.27) & $(169.64)^{\star}$ & $(190.36)^{\star}$ & $(167.73)^{\star}$ & $(168.63)^{\star}$ \\
\hline \multirow[t]{2}{*}{ BMI } & 50.49 & 56.86 & 59.65 & 47.90 & 54.44 & 39.71 & 49.96 & 53.85 & 34.88 \\
\hline & $(23.90)^{\star}$ & $(22.69) \star$ & $(23.86)^{\star}$ & $(23.71)^{\star}$ & $(20.35)^{\star}$ & $(23.94)$ & $(26.87)$ & $(23.66)^{\star}$ & $(23.78)$ \\
\hline \multicolumn{10}{|c|}{$\begin{array}{l}\text { Imaging } \\
\text { classifications }\end{array}$} \\
\hline \multirow[t]{2}{*}{ 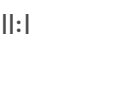 } & -267.83 & -200.72 & -183.17 & -135.00 & -302.68 & -200.10 & -173.83 & -195.91 & -160.55 \\
\hline & $(160.31)$ & $(149.26)$ & $(156.42)$ & $(160.41)$ & $(137.15)^{*}$ & (161.78) & $(180.65)$ & (161.09) & $(161.22)$ \\
\hline \multirow[t]{2}{*}{ III:I } & -29.07 & 2.11 & 38.89 & -72.91 & -82.53 & -68.79 & -229.22 & -33.62 & -32.41 \\
\hline & $(261.51)$ & $(247.77)$ & $(260.78)$ & (259.90) & $(223.31)^{*}$ & $(262.33$ & $(294.37)$ & (259.69) & $(261.00)$ \\
\hline \multicolumn{10}{|c|}{ Random effect } \\
\hline \multirow{2}{*}{$\begin{array}{l}\sigma_{\mathrm{u} 1}^{2}(\mathrm{Time} \\
\text { slope })\end{array}$} & 73174.32 & 43723.62 & 12896.38 & 43618.04 & 41718.29 & 13848.73 & 30966.49 & 8800.04 & 3298.74 \\
\hline & $(16069.15)^{\star}$ & $(10873.24)^{\star}$ & (9931.64) & $(14241.64)^{\star}$ & $(10114.27)^{*}$ & (12615.53) & (16531.10) & (14283.31) & (13403.50) \\
\hline
\end{tabular}

\section{Discussion}

This is the first study to evaluate the dynamic changes in acupoint sensitization. The phenomenon of acupoint sensitization had been more dynamically observed in the present longitudinal study compared with previous studies. Multilevel model analyses were used to comprehensively explore the relationships between various factors and acupoint sensitization.

Previous studies have showed that the PPTs of related acupoints in patients with KOA are lower than those in healthy subjects;[34] however, the dynamic variations in the PPTs of these acupoints over time and in accordance with the patients' conditions are still unclear. The present study found that the PPTs of all acupoints decreased over the 4-week observation period. Without medical intervention, the PPTs of the acupoints reduced as the KOA condition progressed. Based on the results of literature data-mining and a pilot study,[34] the 19 acupoints assessed in the present study had a close relationship with the KOA condition, and so the PPTs of all of the 19 acupoints (13 localized acupoints and six distant acupoints) decreased over time.

The single-factor multilevel model analysis showed that the PPTs of the acupoints decreased with the progression of the clinical stage of KOA; compared with patients with imaging classification I, the PPTs at Xuehai (SP-10), Heding (EX-LE2), Ququan (LR-8), Yingu (KI-10), Xiguan (LR-7), and Qiuxu (GB-40) in patients with imaging classification II and PPTs at Yaoyangguan (DU-3) in patients with imaging classification III and above were significantly decreased. The multifactor multilevel model analysis showed that the PPTs of 11 acupoints decreased significantly with the progression of the clinical stage; compared with patients with imaging classification I, the PPTs at Xuehai (SP-10), Heding (EX-LE2), Ququan (LR-8), Yingu (KI-10), Xiguan (LR-7), and Qiuxu (GB-40) in patients with imaging classification II and the PPTs at Qiuxu (GB-40) in patients with imaging classification III and above were significantly decreased.

The abovementioned findings indicated that acupoints became more sensitive to pain with the progression of the clinical stage of KOA. Some of the 19 acupoints also showed a connection between the PPT and the imaging classification of KOA. In TCM theory, there are close connections between the disease conditions and their respective acupoints, and these points become sensitized when the body is in a diseased state.[28] Acupoints are considered to be 'alive', as they became activated or sensitized in pathological conditions.[29] The states of the acupoints change with the disease condition, as do the PPTs of the acupoints.

Few studies have focused on the relationship between disease severity and acupoint PPTs. Radiographic changes and symptoms, especially pain, are often used in the assessment of KOA.[42] [47] However, for the individual patient with OA, there is often a relatively weak or almost no association between the actual tissue damage and the associated pain intensity, which suggests that pain sensitization develops due to neuroplastic changes rather than actual structural joint damage as the disease progresses.[47] A better index with which to assess the severity of KOA is the clinical stage, comprising a combination of knee joint imaging and comprehensive judgment of clinical symptoms.[42, 43] KOA is a chronic and progressive disease, and obvious changes in the knee joint structure cannot be observed in the short term; therefore, imaging alone is not adequate to evaluate the condition of patients with KOA. However, clinical 
symptoms, mainly comprising pain, are subjective and cannot comprehensively estimate the KOA condition. The actual severity of KOA disease is better reflected by the clinical stage, which varies due to the remission or aggravation of symptoms combined with imaging results.

Sex and BMI influenced the PPTs of the acupoints. Females and patients with a lower BMI were more sensitive to tenderness, and had lower acupoint PPTs. Compared with males with symptomatic knee OA, females with symptomatic knee OA tend to have lower heat, cold, and pressure thresholds/tolerances, and greater temporal summation of pain.[48] The thickness of subcutaneous fat might influence the acupoint PPTs, making patients with a lower BMI more sensitive to pain.

It is effective to analyze the ordered category data with repeated measurements in a multilevel model. In the multi-factor multilevel model analysis, the PPTs decreased more with the progression of the clinical stage of KOA at 12 acupoints in the random effects model and 11 acupoints in the fixed effects model. The following eight acupoints showed significant PPT changes in accordance with the KOA clinical stage in both effect models: Liangqiu (ST-34), Dubi (ST35), Weiyang(BL-39), Yinglingquan (SP-9), Xiguan (LR-7), Zusanli (ST-36), Yanglingquan (GB-34), and Qiuxu (GB-40). Therefore, these eight acupoints may be most associated with the KOA severity. Stimulation of these acupoints may achieve optimal clinical outcomes in patients with KOA.

This study had two limitations. First, 4 weeks might be not long enough to observe pathological changes. As the treatments of the patients were limited during the observation period, we set the observation period as 4 weeks in consideration of the patients' conditions and ethical issues. Second, although acupuncture treatment and some pharmacotherapies were not allowed during the 4-week observation period, other pharmacotherapy or non-pharmacotherapy treatments (except for acupuncture) and self-adjustment were not recorded. It is unclear whether this impacted the results, which might lead to certain bias. A longer observation period and more comprehensive research record are needed in further study.

\section{Conclusions}

The related acupoints of patients with KOA became more pain-sensitive over time, and the PPTs of the related acupoints were closely related to the severity of the disease. Among the 19 assessed acupoints, Liangqiu (ST-34), Dubi (ST-35), Weiyang (BL-39), Yinglingquan (SP-9), Xiguan (LR-7), Zusanli (ST-36), Yanglingquan (GB-34), and Qiuxu (GB-40) were the most closely related to the disease severity, which suggests to stimulate these 8 acupoints to obtain the optimal therapeutic effects.

\section{Abbreviations}

$\mathrm{OA}=$ osteoarthritis, $\mathrm{KOA}=$ knee osteoarthritis, $\mathrm{PPT}=$ pressure pain threshold, $\mathrm{TCM}=$ traditional Chinese medicine, $\mathrm{BMI}=$ body mass index, no. $=$ number .

\section{Declarations}

Data Availability All data included in this study are available upon request from the corresponding author on reasonable request.

Acknowledgment We acknowledge the help and contributions from the subjects, investigators, and experts in each center. We thank Kelly Zammit, BVSc, from Liwen Bianji, Edanz Editing China (www.liwenbianji.cn/ac), for editing the English text of a draft of this manuscript.

Author Contributions FRL conceived the study and acquired the funding. GYG, YY, MSS and ZHY contributed equally to this work. GYG wrote the draft. YY analyzed the data. GYG, MSS, ZHY and LJL performed data collection. ZH, CJ, DJC, FRL, ZL and CXY designed the study and modified the manuscript. All the authors discussed, read and revised the manuscript, and gave final approval for the publication of this paper.

ORCID iD Guoyan Geng ORCID: 0000-0002-0730-2170

Funding This work was financially supported by the National Natural Science Foundation of China (numbers 81590951).

Competing interests None declared.

Patient consent Obtained.

Ethics approval The study protocol has been approved by the institutional review board and ethics committee of the First Affiliated Hospital of Chengdu University of Traditional Chinese Medicine (September 2016) (approval number: 2016KL-041).

Provenance and peer review Not commissioned; externally peer reviewed.

Consent for publication Not applicable.

\section{References}

1. Hunter DJ, Bierma-Zeinstra S: Osteoarthritis. The Lancet 2019, 393(10182):1745-1759.

2. Daniel PA, Andrew J, M Kassim J, Cyrus C, Adolfo DP, Arden NK: Incidence and risk factors for clinically diagnosed knee, hip and hand osteoarthritis: influences of age, gender and osteoarthritis affecting other joints. Annals of the Rheumatic Diseases 2014, 73(9):1659-1664.

3. Marita C, Emma S, Damian H, Sandra N, llana A, Marlene F, Lisa B, Sean W, Francis G, Hill CL: The global burden of hip and knee osteoarthritis: estimates from the global burden of disease 2010 study. Annals of the Rheumatic Diseases 2014, 73(7):1323.

4. Hunter DJ, Deborah S, Emily C: The individual and socioeconomic impact of osteoarthritis. Nature Reviews Rheumatology 2014, 10(7):437-441. 
5. Salter DM, Su SL, Lee HS: Epidemiology and genetics of osteoarthritis. Journal of Medical Sciences 2014, 34(6):252-259.

6. Hauk L: Treatment of knee osteoarthritis: a clinical practice guideline from the AAOS. Am Fam Physician 2014, 89(11):918-920.

7. Tang X, Wang S, Zhan S, Niu J, Tao K, Zhang Y, Lin J: The Prevalence of Symptomatic Knee Osteoarthritis in China: Results From the China Health and Retirement Longitudinal Study. Arthritis Rheumatol 2016, 68(3):648-653.

8. Peat G, ., Mccarney R, ., Croft $\mathrm{P}$. . Knee pain and osteoarthritis in older adults: a review of community burden and current use of primary health care. Annals of the Rheumatic Diseases 2001, 60(2):91-97.

9. Nelson AE, Allen KD, Golightly YM, Goode AP, Jordan JM: A systematic review of recommendations and guidelines for the management of osteoarthritis: The chronic osteoarthritis management initiative of the U.S. bone and joint initiative. Semin Arthritis Rheum 2014, 43(6):701-712.

10. McAlindon TE, Bannuru RR, Sullivan MC, Arden NK, Berenbaum F, Bierma-Zeinstra SM, Hawker GA, Henrotin Y, Hunter DJ, Kawaguchi H et al: OARSI guidelines for the non-surgical management of knee osteoarthritis. Osteoarthritis Cartilage 2014, 22(3):363-388.

11. da Costa BR, Reichenbach S, Keller N, Nartey L, Wandel S, Jüni P, Trelle S: Effectiveness of non-steroidal anti-inflammatory drugs for the treatment of pain in knee and hip osteoarthritis: a network meta-analysis. The Lancet 2017, 390(10090):e21-e33.

12. Martel-Pelletier J, Barr AJ, Cicuttini FM, Conaghan PG, Cooper C, Goldring MB, Goldring SR, Jones G, Teichtahl AJ, Pelletier J-P: Osteoarthritis. Nature Reviews Disease Primers 2016, 2(1):16072.

13. Krebs EE, Gravely A, Nugent S, Jensen AC, DeRonne B, Goldsmith ES, Kroenke K, Bair MJ, Noorbaloochi S: Effect of Opioid vs Nonopioid Medications on Pain-Related Function in Patients With Chronic Back Pain or Hip or Knee Osteoarthritis Pain: The SPACE Randomized Clinical Trial. JAMA 2018, 319(9):872-882.

14. Collaboration* CatNTC: Vascular and upper gastrointestinal effects of non-steroidal anti-inflammatory drugs: meta-analyses of individual participant data from randomised trials. The Lancet 2013, 382(9894):769-779.

15. Schmeltzer PA, Kosinski AS, Kleiner DE, Hoofnagle JH, Stolz A, Fontana RJ, Russo MW: Liver Injury from Nonsteroidal Anti-inflammatory Drugs in the United States. Liver International 2016, 36(4):603-609.

16. Mao JJ, Farrar JT, Deborah B, Jarcy Z, Marjorie B, Christina S, Angela DM, Xie SX: Electroacupuncture for fatigue, sleep, and psychological distress in breast cancer patients with aromatase inhibitor-related arthralgia: a randomized trial. Cancer 2015, 120(23):3744-3751.

17. Hershman DL, Unger JM, Greenlee H, Capodice JL, Crew KD: Effect of Acupuncture vs Sham Acupuncture or Waitlist Control on Joint Pain Related to Aromatase Inhibitors Among Women With Early-Stage Breast Cancer: A Randomized Clinical Trial. Jama the Journal of the American Medical Association 2018, 320(2):167.

18. Zhao L, Li D, Zheng H, Chang X, Cui J, Wang R, Shi J, Fan H, Li Y, Sun X et al: Acupuncture as Adjunctive Therapy for Chronic Stable Angina: A Randomized Clinical Trial. JAMA Intern Med 2019

19. Zhao L, Chen J, Li Y, et al.: The long-term effect of acupuncture for migraine prophylaxis: A randomized clinical trial. JAMA Internal Medicine 2017, 177(4):508-515.

20. MacPherson H, Vertosick EA, Foster NE, Lewith G, Linde K, Sherman KJ, Witt CM, Vickers AJ, Acupuncture Trialists C: The persistence of the effects of acupuncture after a course of treatment: a meta-analysis of patients with chronic pain. Pain 2017, 158(5):784-793.

21. Chen N, Wang J, Mucelli A, Zhang X, Wang C: Electro-Acupuncture is Beneficial for Knee Osteoarthritis: The Evidence from Meta-Analysis of Randomized Controlled Trials. Am J Chin Med 2017, 45(5):965-985.

22. Li J, Li YX, Luo LJ, Ye J, Zhong DL, Xiao QW, Zheng H, Geng CM, Jin RJ, Liang FR: The effectiveness and safety of acupuncture for knee osteoarthritis: An overview of systematic reviews. Medicine (Baltimore) 2019, 98(28):e16301.

23. Que BF, Zeng XL, Wu WB, Lian QQ, Tang YL, Liu TS, Qiu R: Analgesic and warming meridians as well as unblocking collateral effects of needle warming moxibustion in patients with osteoarthritis of the knee joint. International Journal of Clinical and Experimental Medicine 2018, 11(9):9966-9973.

24. Corbett MS, Rice SJ, Madurasinghe V, Slack R, Fayter DA, Harden M, Sutton AJ, Macpherson H, Woolacott NF: Acupuncture and other physical treatments for the relief of pain due to osteoarthritis of the knee: network meta-analysis. Osteoarthritis Cartilage 2013, 21(9):1290-1298.

25. Schiphof D, van den Driest JJ, Runhaar J: Osteoarthritis year in review 2017: rehabilitation and outcomes. Osteoarthritis and Cartilage 2018, 26(3):326340.

26. Hinman R, McCrory P, Pirotta M, Relf I, Forbes A, Crossley K, Williamson E, Kyriakides M, Novy K, Metcalf B et al: Acupuncture for Chronic Knee Pain A Randomized Clinical Trial. JAMA 2014, 312:1313-1322.

27. Vickers AJ, Vertosick EA, Lewith G, MacPherson H, Foster NE, Sherman KJ, Irnich D, Witt CM, Linde K: Acupuncture for Chronic Pain: Update of an Individual Patient Data Meta-Analysis. J Pain 2018, 19(5):455-474.

28. Zhu B: [The plasticity of acupoint]. Zhongguo Zhen Jiu 2015, 35(11):1203-1208.

29. Chen R, Kang M, Chen M: [Retum of Qibo: on hypothesis of sensitization state of acupoints]. Zhongguo Zhenjiu 2011, 31(2).

30. Zhu B: [The sensitization phenomenon of acupoint and biological significances]. zhongguo zhenjiu 2019, 39(02):115-121.

31. Ben H, Li L, Rong PJ, Jin ZG, Zhang JL, Li YH, Li X: Observation of Pain-Sensitive Points along the Meridians in Patients with Gastric Ulcer or Gastritis. Evid Based Complement Alternat Med 2012, 2012:130802.

32. Shi J, Wang J, Wang Y, Liu K, Fu Y, Sun JH, Zhao JP, Shao XM, Feng SF, Yang YW et al: [Correlation Between Referred Pain Region and Sensitized Acupoints in Patients with Stable Angina Pectoris and Distribution of Sensitized Spots in Rats with Myocardial Ischemia]. Zhen Ci Yan Jiu 2018, 43(5):277-284 
33. Yan CQ, Zhang S, Li QQ, Zhang LW, Wang XR, Fu QN, Shi GX, Liu CZ: Detection of peripheral and central sensitisation at acupoints in patients with unilateral shoulder pain in Beijing: a cross-sectional matched case-control study. BMJ Open 2017, 7(6):e014438.

34. Luo Y-N, Zhou Y-M, Zhong X, Zhao L, Zheng Q-H, Zheng H, Tang L, Jia P-L, Wu Q, Huang C et al: Observation of pain-sensitive points in patients with knee osteoarthritis: A pilot study. European Journal of Integrative Medicine 2018, 21:77-81.

35. Persson AL, Brogårdh C, Sjölund BH: Tender or not tender: test-retest repeatability of pressure pain thresholds in the trapezius and deltoid muscles of healthy women. Journal of Rehabilitation Medicine 2004, 36(1):17.

36. Sun M, Geng G, Chen J, Ma X, Yan M, Liu X, Du J, Cai D, Zheng H, Zhao L et al: Acupuncture for chronic neck pain with sensitive points: study protocol for a multicentre randomised controlled trial. BMJ Open 2019, 9(7):e026904.

37. Sun M, Tao S, Geng G, Peng J, Ma X, Yan M, Chen J, Cai D, Zheng H, Yang C et al: Identification of the optimal points for the acupuncture treatment of neck pain in China: protocol for a multicenter, matched, case-control study. BMJ Open 2019, 9(8):e029194.

38. von Elm E, Altman DG, Egger M, Pocock SJ, Gøtzsche PC, Vandenbroucke JP: Strengthening the Reporting of Observational Studies in Epidemiology (STROBE) statement: guidelines for reporting observational studies. Bmj 2007, 335(7624):806-808.

39. Association OGoCO: Guideline for diagnosis and treatment of osteoarthritis. Chinese Journal of Joint Surgery (Electronic Edition) 2007, 1(4):287-291.

40. Bijlsma JW, Berenbaum F, Lafeber FP: Osteoarthritis: an update with relevance for clinical practice. Lancet 2011, 377(9783):2115-2126.

41. National Clinical Guideline C: National Institute for Health and Clinical Excellence: Guidance. In: Osteoarthritis: Care and Management in Adults. edn. London: National Institute for Health and Care Excellence (UK) Copyright (c) National Clinical Guideline Centre, 2014.; 2014.

42. Braun HJ, Gold GE: Diagnosis of osteoarthritis: imaging. Bone 2012, 51(2):278-288.

43. Liu K, Ling L, Hu H: [Degenerative process and diagnosis stage of osteoarthritis]. Chinese Journal of Joint Surgery(Electronic Edition) 2017, 11(4):97-100. 44. Diggle P, Heagerty P, Liang KY, Zeger S: Analysis of Longitudinal Data, vol. 90, Second edn. Oxford: Oxford University Press; 2002.

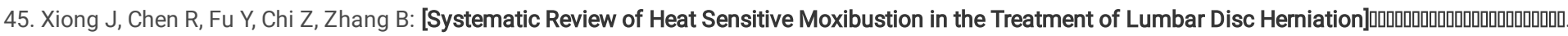
Jiang Xi Zhong Yi Yao 2011, 42(3):48-51.

46. Fan Li, Xu Zhenhua, Xiaoping Z: [Clinical study on the treatment of chronic lumbar muscle strain with heat sensitive moxibustion] Zhong Yi Xue Yuan Xue Bao 2007, 019(5):61-62.

47. Arendt-Nielsen L: Pain sensitisation in osteoarthritis. Clinical \& Experimental Rheumatology 2017, 35(5):S68-S74.

48. Bartley EJ, King CD, Sibille KT, Cruz-Almeida Y, Glover TL, Goodin BR, Sotolongo AS, Herbert MS, Bulls HW: Enhanced Pain Sensitivity Among Individuals With Symptomatic Knee Osteoarthritis: Potential Sex Differences in Central Sensitization. Arthritis Care \& Research 2016, 68(4):472-480.

\section{Figures}
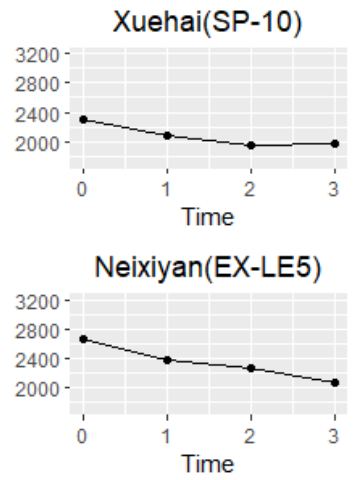

Yanglingquan(GB-34)

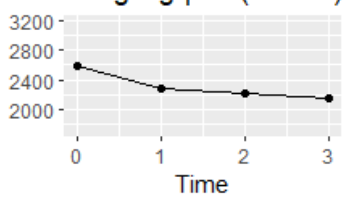

$\operatorname{Dazhu(BL-11)}$

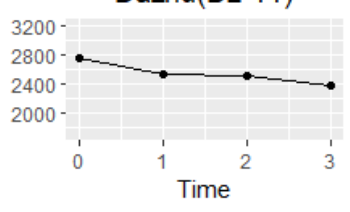

Figure 1

PPTs (gf) of the 19 acupoints at each of the four measurement timepoints
Liangqiu(ST-34)

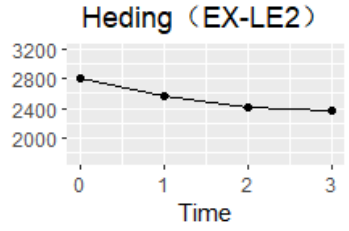

Dubi(ST-35)

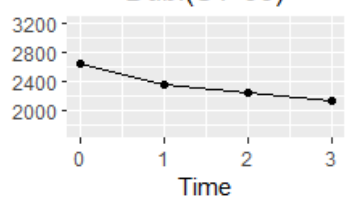

Weiyang(BL-39)

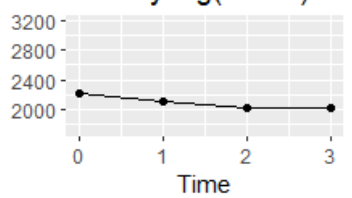

Shenshu(BL-23)

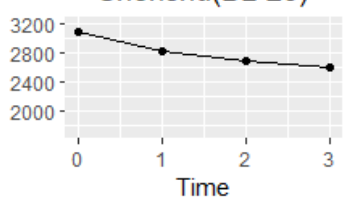

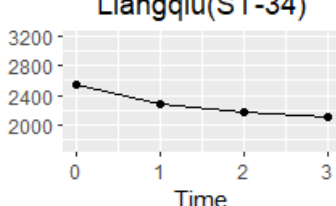

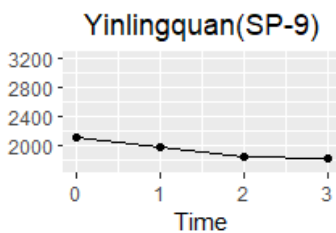

Weizhong(BL-40)
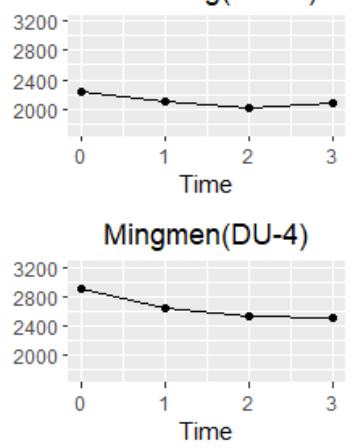
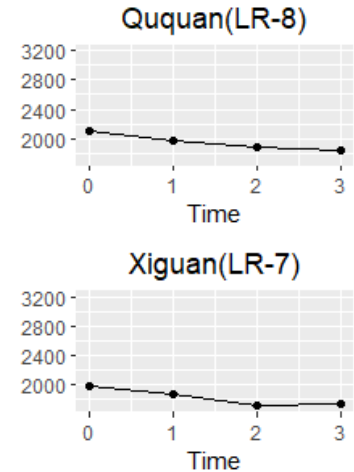

Xuanzhong(GB-39)

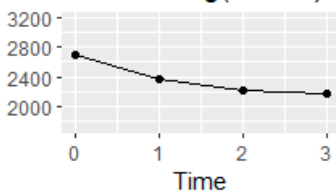

Yaoyangguan(DU-3)

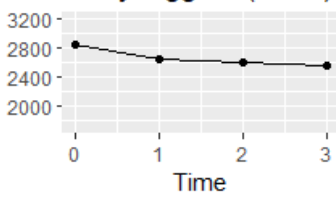

Yingu(KI-10)

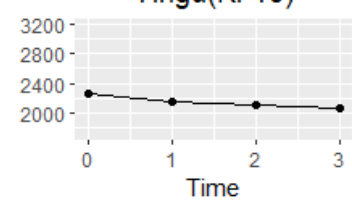

Zusanli(ST-36)

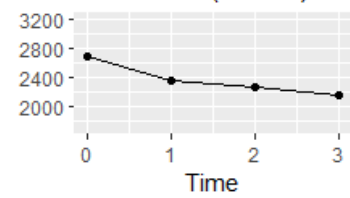

Qiuxu(GB-40)

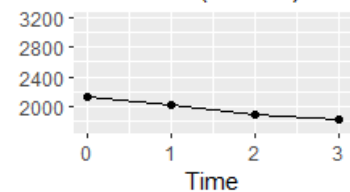

Page 14/14 